\title{
REVIEW
}

\section{Metastatic renal cell cancer}

\author{
Finn Rasmussen \\ Department of Radiology, Aarhus University Hospital, Denmark \\ Corresponding address: Finn Rasmussen, MD, MDSc, Department of Radiology, Aarhus University Hospital, \\ Nørrebrogade 44, Aarhus 8000, Denmark. \\ Email: firasmerm. dk
}

\begin{abstract}
Targeted therapy is the treatment of choice in patients with metastatic renal cell cancer (mRCC) at most institutions although a combination of cytokine therapy and targeted therapy still is being investigated. Morphological size-based criteria (RECIST) has failed in monitoring the effect of targeted therapy in patients with mRCC, as successful therapy often does not result in a decrease in tumour size. Modifications of size-based criteria and criteria based on computed tomography (CT) contrast enhancement has been introduced. Different imaging modalities that rely on characteristics other than size such as dynamic contrast-enhanced (DCE) ultrasonography, DCE CT, DCE magnetic resonance imaging (MRI), diffusion-weighted MRI, positron emission tomography and texture analysis seem to contribute with prognostic information, even at baseline scans, and can predict tumour response early after initiating therapy. No new standard for the imaging follow-up of targeted therapy in $\mathrm{mRCC}$ has been established.
\end{abstract}

Keywords: Targeted therapy; renal cell carcinoma; mRCC.

\section{Introduction}

Renal cell carcinoma (RCC) accounts for $2 \%$ of all adult malignancies, and represents $90 \%$ of renal tumours, of which $75 \%$ are clear-cell carcinomas ${ }^{[1,2]}$. Approximately $30 \%$ of patients present with metastatic disease at the time of diagnosis, and approximately $30 \%$ of patients with initially localized disease ultimately develop metastatic disease. Untreated, the 5-year survival rate for metastatic RCC (mRCC) is $2 \%$, and the median survival is approximately 8 months ${ }^{[2,3]}$.

As a result of the increased use of diagnostic imaging performed for other indications, an increasing number of RCCs are found incidentally, so the size of the tumour and the stage at presentation are currently decreasing ${ }^{[2,4]}$.

RCC is remarkably resistant to conventional chemotherapy and radiotherapy. Before the era of targeted therapies, cytokine therapy consisting of interleukin-2 and interferon were standard therapies for mRCC. However, the development of targeted agents has changed the treatment of choice for patients with $\mathrm{mRCC}^{[1,3]}$.

Objective response assessment is important to describe the effect of anticancer treatment. For years, it was generally accepted that a decrease in tumour size based on morphological imaging correlated with treatment effect. However, targeted therapies frequently cause disease stabilization rather than a substantial regression in tumour size, and early necrosis induced by targeted therapies may even simulate progressive disease ${ }^{[5]}$.

To reduce costs and drug-induced toxicities from the growing number of targeted agents, there is a need for imaging techniques that can predict tumour response or resistance, optimally in the pretreatment phase or as early as possible after initiating therapy.

\section{Morphological imaging}

Since 2000, the Response Evaluation Criteria in Solid Tumours (RECIST) has become the most commonly used international guideline for evaluation of treatment response in solid tumours ${ }^{[6]}$. RECIST is a one-dimensional measurement of the sum of the longest diameters of target lesions. Often the initial response of targeted therapy in $\mathrm{mRCC}$ is associated with tumour stabilization and morphological changes rather than a shrinkage in tumour size (Fig. 1), and the best response according to RECIST may be postponed until several months after initial drug administration ${ }^{[7]}$. Using RECIST in the follow-up of patients with $\mathrm{mRCC}$ receiving sunitinib, stable disease at first evaluation is not predictive for progressive disease or partial response at a later 
(a)
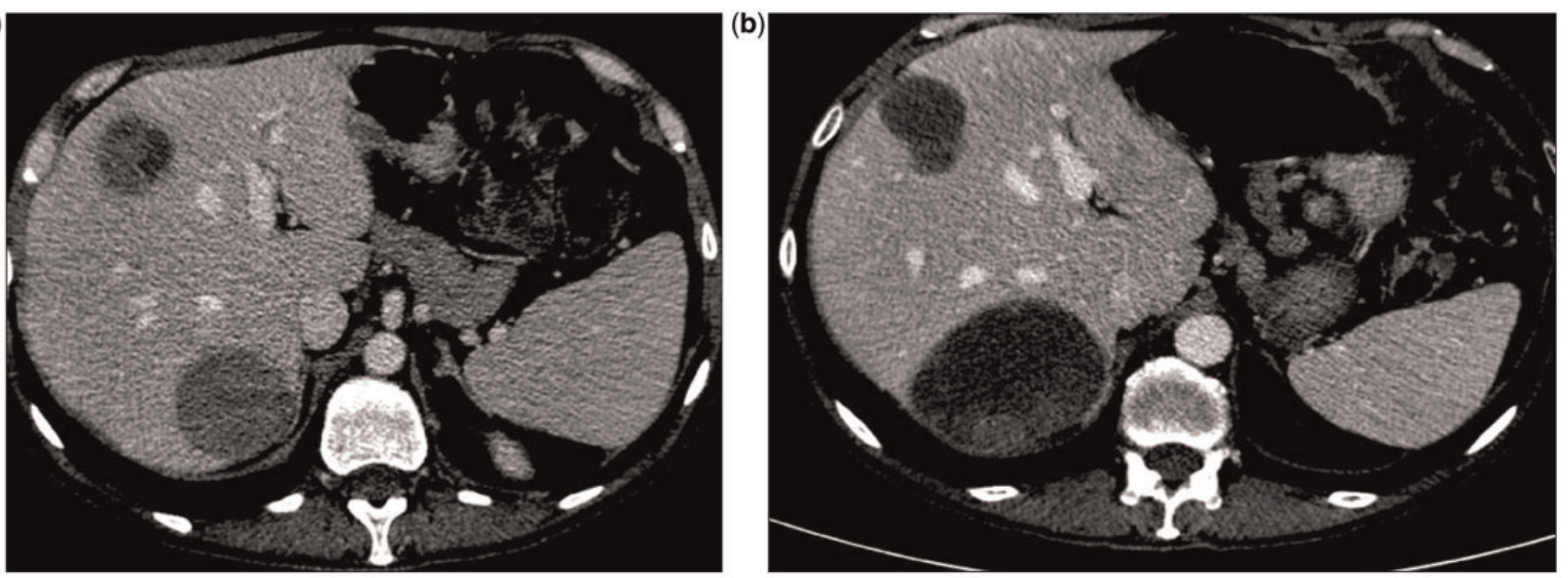

(c)
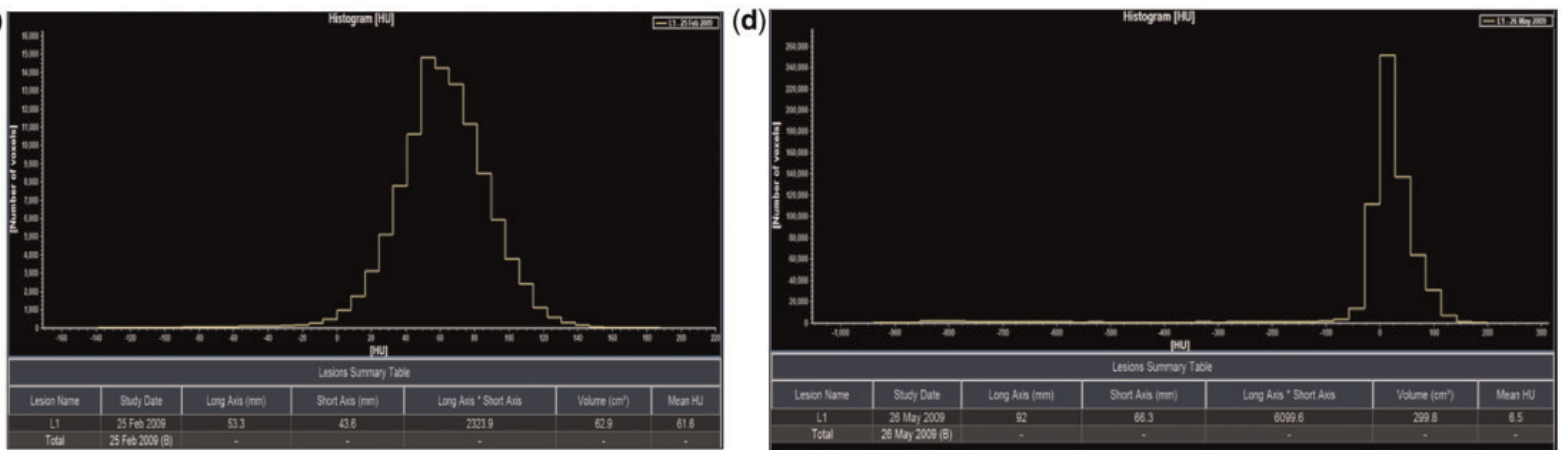

Figure 1 Liver metastases in a patient with renal cell cancer (a) before and (b) after sunitinib therapy. Semi-automatic lesion segmentation can be performed and quantitative data derived, (c) before and (d) after. The diameters, volume, mean attenuation of the whole lesion and histograms showing the distribution of voxels with a certain attenuation value can be displayed. In the liver lesion shown, it is obvious that the diameters and the volume of the lesion increased. The mean attenuation of the lesion decreased and the histogram changed dramatically. Instead of size measurements and mean attenuation, alterations in the histogram may be a more sensitive method for assessing treatment effect.

evaluation $^{[8]}$. In another study on patients with mRCC treated with sunitinib using progression-free survival as the end point, Thiam et al. ${ }^{[9]}$ reported that a threshold of at least $10 \%$ decrease in the sum of the longest diameters was more accurate in predicting partial response than the threshold of $30 \%$ as defined in RECIST. Krabejewski et al. ${ }^{[10]}$ verified in a recent retrospective study that a $10 \%$ reduction in the sum of the longest diameters on the first follow-up computed tomography (CT) scan is a predictor of a positive outcome in patients with $\mathrm{mRCC}$ receiving targeted therapy. So it seems that when using lesion size for treatment assessment, a threshold of $-10 \%$ instead of $-30 \%$ is better at distinguishing responders from non-responders among patients with $\mathrm{mRCC}$ receiving targeted therapy.

RCC tumours are highly perfused, and tumour enhancement on contrast-enhanced CT is correlated with microvessel density (a pathologic marker representing tumour vascularity in $\mathrm{RCC})^{[11]}$ and tumour angiogenesis $^{[12]}$. A decrease in tumour density is associated with response to therapy and the development of necrotic areas ${ }^{[13]}$. Therefore, application of contrast-enhanced CT according to the Choi criteria ${ }^{[14]}$ seems attractive.
The Choi criteria prescribe partial response as more than $10 \%$ decrease in one-dimensional tumour size or a $15 \%$ decrease in tumour attenuation on contrast-enhanced CT. Progressive disease is defined as more than $10 \%$ increase in size while not meeting the partial response criteria for change in enhancement.

At first evaluation for patients with $\mathrm{mRCC}$ treated with targeted therapy, assessment using the Choi criteria has been shown to have better predictive value for progression-free survival (PFS) and overall survival (OS) than RECIST $^{[8,15]}$. However, it has also been shown that a $10 \%$ reduction in the sum of largest diameters is a better predictor than the Choi criteria ${ }^{[10]}$. Using modified Choi criteria (a decrease in size $>10 \%$ and a decrease in tumour attenuation $>15 \%$ ), Nathan et al. ${ }^{[16]}$ showed that such estimation is better correlated to time to progression than the Choi criteria. When contrast attenuation-based criteria are applied, standardization of the contrast phases is crucial. Enhancement is not only influenced by the contrast media phase, but also by cardiac output, distribution volume, injection rate, volume and concentration of the contrast medium and the acquisition parameters of the scanner. Different scanners may 
give different results despite using the same acquisition parameter settings and the same contrast media policy.

In a retrospective study assessing tumour enhancement on arterial phase scans, Han et al. ${ }^{[17]}$ found that pretreatment tumour enhancement was associated with treatment outcomes after targeted therapy in patients with mRCC. It has also been demonstrated that an increase in contrast enhancement may be associated with progressive disease ${ }^{[18]}$.

Smith et al. ${ }^{[18]}$ introduced the size and attenuation CT (SACT) criteria, and found in a retrospective study of patients with $\mathrm{mRCC}$ receiving targeted therapy that these criteria gave better prognostic information than the Choi criteria or the RECIST criteria. In the SACT criteria, three-dimensional volumetric attenuation assessment was combined with tumour size. Due to the fact that central necrosis is often seen in responders, the same authors incorporated this in morphology, attenuation, size and structure (MASS) criteria ${ }^{[19]}$, and found that the assessment of mRCC target lesions on contrastenhanced CT is more accurate using MASS criteria than SACT criteria, modified Choi criteria and RECIST criteria. Although promising, the MASS criteria need to be prospectively validated in larger patient populations.

Although semi-automatic tools make it easy to segment and measure the volume of a lesion and calculate the mean attenuation of a whole lesion (SACT criteria), there is as yet no evidence that three-dimensional measurements are better than two-dimensional measurements. However, as seen in Fig. 1, a histogram showing the distribution of voxels according to Hounsfield unit values can also be derived from three-dimensional measurements. Such histograms show that the tumour does have heterogeneous attenuations and that the histogram and the heterogeneity may change due to treatment. Using CT texture analysis, Goh et al. ${ }^{[20]}$ showed that lesion values of entropy decreased and lesion values of uniformity increased after two cycles of tyrosine kinase inhibitors in mRCC. Furthermore, it was shown that baseline entropy and uniformity correlated with time to progression, suggesting that baseline heterogeneity may additionally present predictive information.

The lung is the most common site for metastases from RCC. Monitoring the effect of treatment of such lesions is a challenge for the aforementioned criteria (Fig. 2), and therefore some criteria definitions omit lung metastases. Lung metastasis in $\mathrm{mRCC}$ quite often cavitate and contain air.

\section{Functional imaging approach}

RCC and its metastases are highly vascular, and targeted therapies use several cellular pathways to interfere with angiogenesis and tumour vasculature. Therefore, imaging modalities that can assess the vascularity and the changes in vascularity of such tumours are of great interest ${ }^{[11,12]}$.

\section{Dynamic contrast-enhanced CT}

Dynamic contrast-enhanced (DCE) CT measures the temporal changes in tissue enhancement after administration of iodinated contrast media. The contrast enhancement is linearly proportional to the iodine concentration. The method can estimate parameters of first-pass perfusion as well as permeability. A positive correlation between microvessel density and the DCE CT measures of relative blood flow and relative blood volume has been shown $^{[17,21,22]}$, and it was found that tumour blood flow and tumour blood volume decreased in mRCC metastases after one cycle of treatment with targeted drugg ${ }^{[23]}$. In our experience, functional parameters such as blood flow and blood volume are superior to measurements of diameter, volume and contrast enhancement (Fig. 3) when early response evaluation is considered using PFS or OS as end points. $\mathrm{Ng}$ et al. ${ }^{[24]}$ demonstrated that tumour blood flow at baseline was inversely correlated to PFS in patients with $\mathrm{mRCC}$ receiving interferon treatment. However, Fournier et al. ${ }^{[23]}$ found that DCE CT baseline figures may predict the response of targeted therapy in mRCC. This discrepancy may rely on different treatment regimens. In our experience, a high baseline blood flow or blood volume is predictive of favourable outcome when PFS or OS are end points. Just as in contrast-enhanced CT, standardization of protocols is necessary when DCE CT is considered ${ }^{[25]}$ before the discipline can be introduced into daily routine, because the results obtained depend on the acquisition parameters and the contrast media policy. However, DCE CT can easily be combined with contrast-enhanced CT of the thorax, abdomen and pelvis, which is the traditional discipline in the routine imaging procedure.

\section{Magnetic resonance imaging}

DCE magnetic resonance imaging (MRI) can also be applied to measure the temporal changes in enhancement after administration of gadolinium-based contrast media. The technique is more complicated than DCE CT, and the relationship between contrast concentration and signal intensity is more complex and non-linear. However, the contrast medium kinetic is similar to iodinated contrast media in CT. Traditionally, parameters such as $K_{\text {trans }}$ are used in DCE MRI, and not first-pass parameters as in CT. $K_{\text {trans }}$ reflects several aspects of the biological response of the tumour to targeted therapy. Flaherty et al. ${ }^{[26]}$ has shown that $K_{\text {trans }}$ decreased significantly after sorafenib treatment in patients with $\mathrm{mRCC}$, and that high $K_{\text {trans }}$ values before treatment were associated with improved PFS. Hahn et al. ${ }^{[27]}$ also showed a correlation between high baseline $K_{\text {trans }}$ values and improved PFS. As with DCE CT, DCE MRI techniques also need standardization before they can be applied in 
(a)

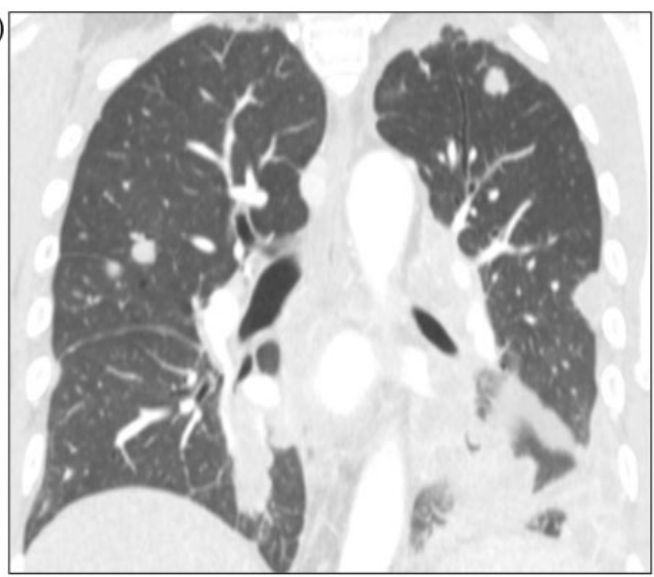

(c)

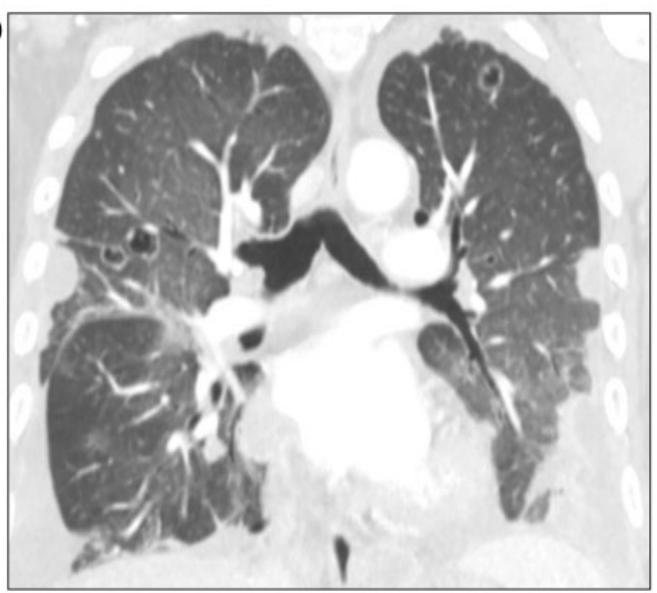

b)

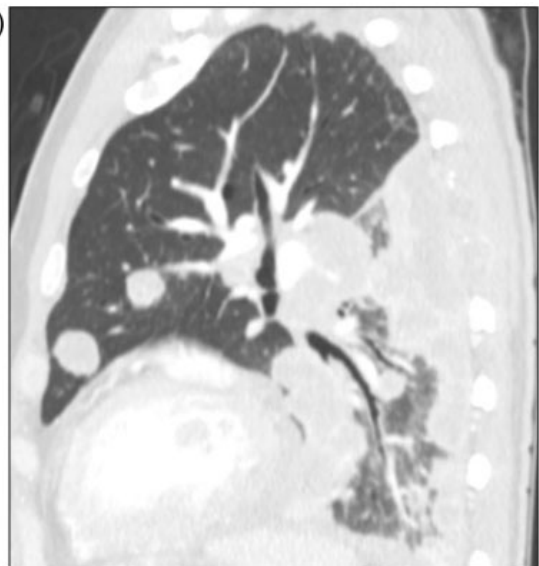

d)

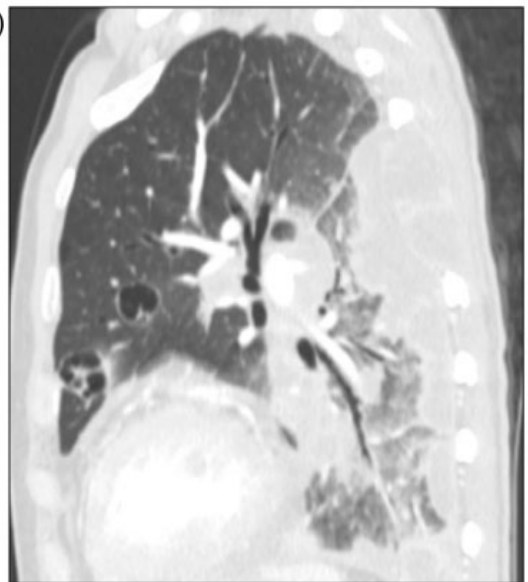

Figure 2 Lung metastases in a patient with renal cell carcinoma (a, b) before and (c, d) after targeted therapy. The most common site for metastases from RCC is the lung. Monitoring the effect of treatment on such lesions is a challenge, and some treatment evaluation criteria even omit lung metastases as target lesions.

daily routine, as the parameters obtained depend on acquisition protocols as well as on consensus of the kinetic model to be used.

Diffusion-weighted (DW) MRI is based on the water diffusion properties of tissues. The technique does not need administration of contrast media. In general, single-shot echo planar imaging sequences based on gradient-echo techniques are used. The strength and duration of the applied diffusion sensitizing gradients is indicated by their $b$-value. The apparent diffusion coefficient (ADC) can then be calculated. Tissues with high cellularity or decreased interstitial spaces show restricted diffusion that results in low ADC values compared with tissues with low cellularity or increased interstitial space. DW MRI has been shown to be helpful in the characterization of renal tumours ${ }^{[28]}$. DW MRI may have the potential to monitor the effect of targeted therapy in mRCC. In a pilot study of 10 patients with mRCC, Desar et al. ${ }^{[29]}$ showed that, compared with baseline, the ADC values increased 3 days after sunitinib treatment followed by a decrease to baseline levels at day 10. Further studies and larger patient groups are needed to evaluate if the early changes observed by
DW MRI can be clinically useful for evaluating the treatment effect of targeted therapy in mRCC.

\section{DCE ultrasonography}

DCE ultrasonography is able to visualize tumour vascularization. Most often a bolus injection of microbubbles is used. The microbubbles serves as a blood pool agent, and time-intensity curve analysis can be applied. Time to peak intensity and area under the curve, which correlate with blood flow and blood volume, can be quantified. In patients with mRCC treated with sunitinib, Laussau et al. ${ }^{[30]}$ showed that DCE ultrasonography parameters at baseline and on day 15 were significantly different between responders and non-responders. DCE ultrasonography does have a major drawback as the discipline cannot evaluate lesions localized to the lung, the most common site of metastases in RCC.

\section{Positron emission tomography}

In positron emission tomography (PET), radiopharmaceuticals are administered. These tracers contain 
(a)

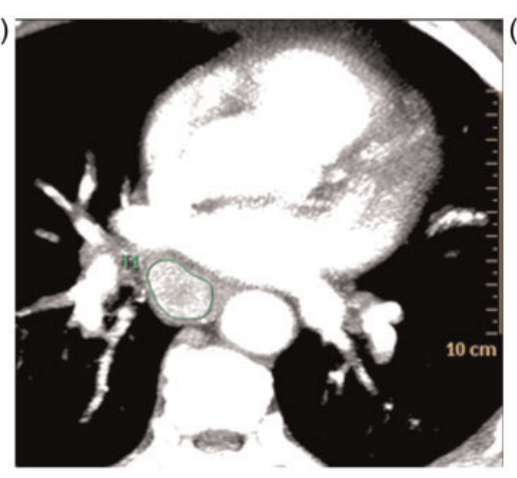

(d)

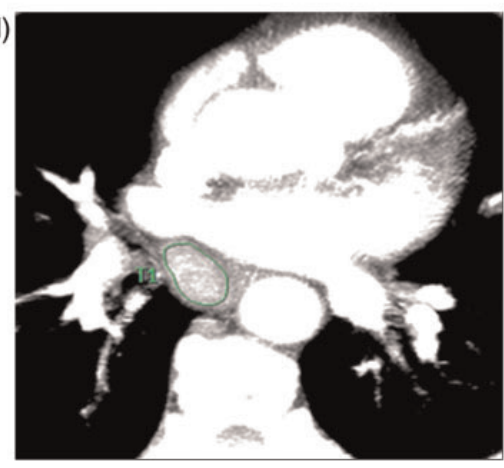

(g)

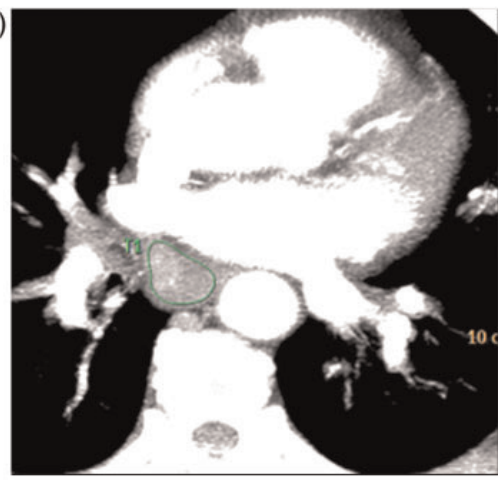

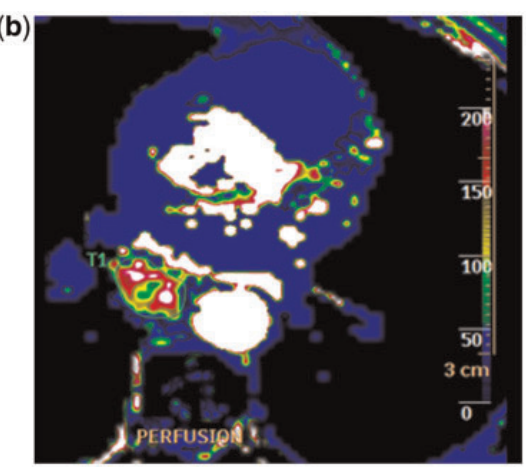

(e)
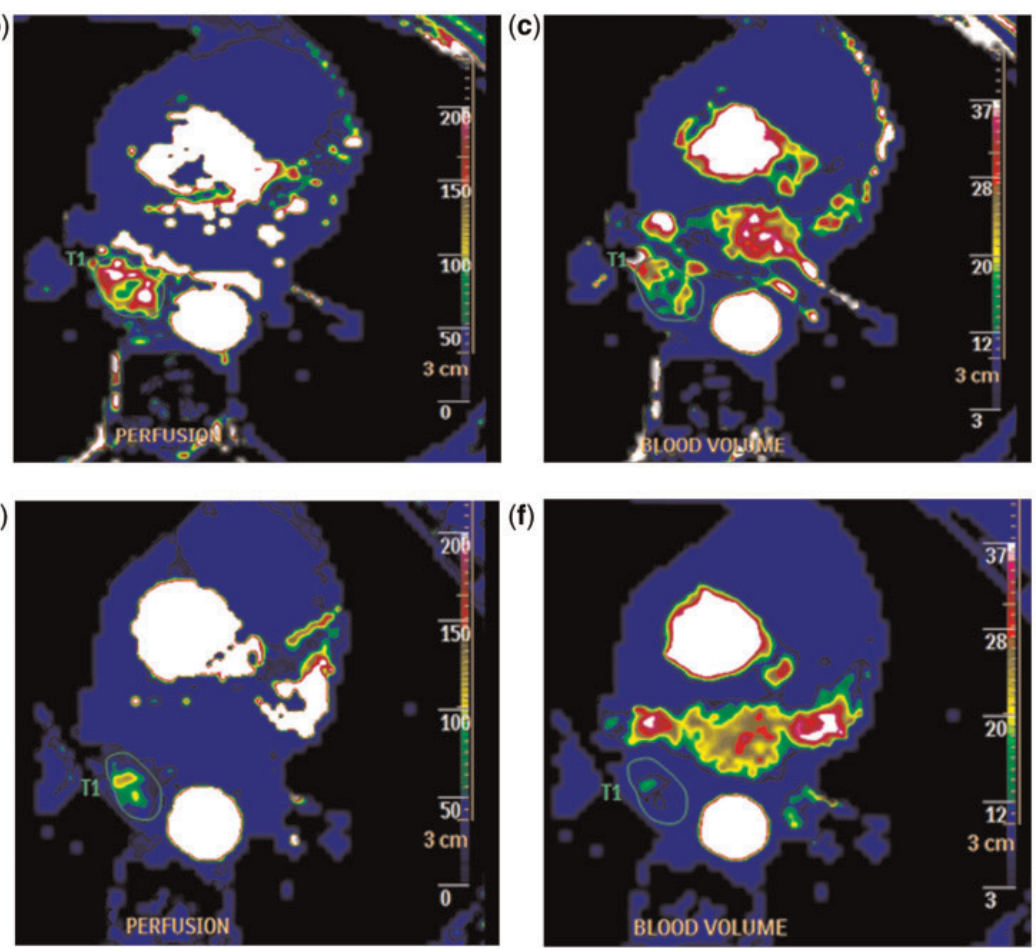

(f)
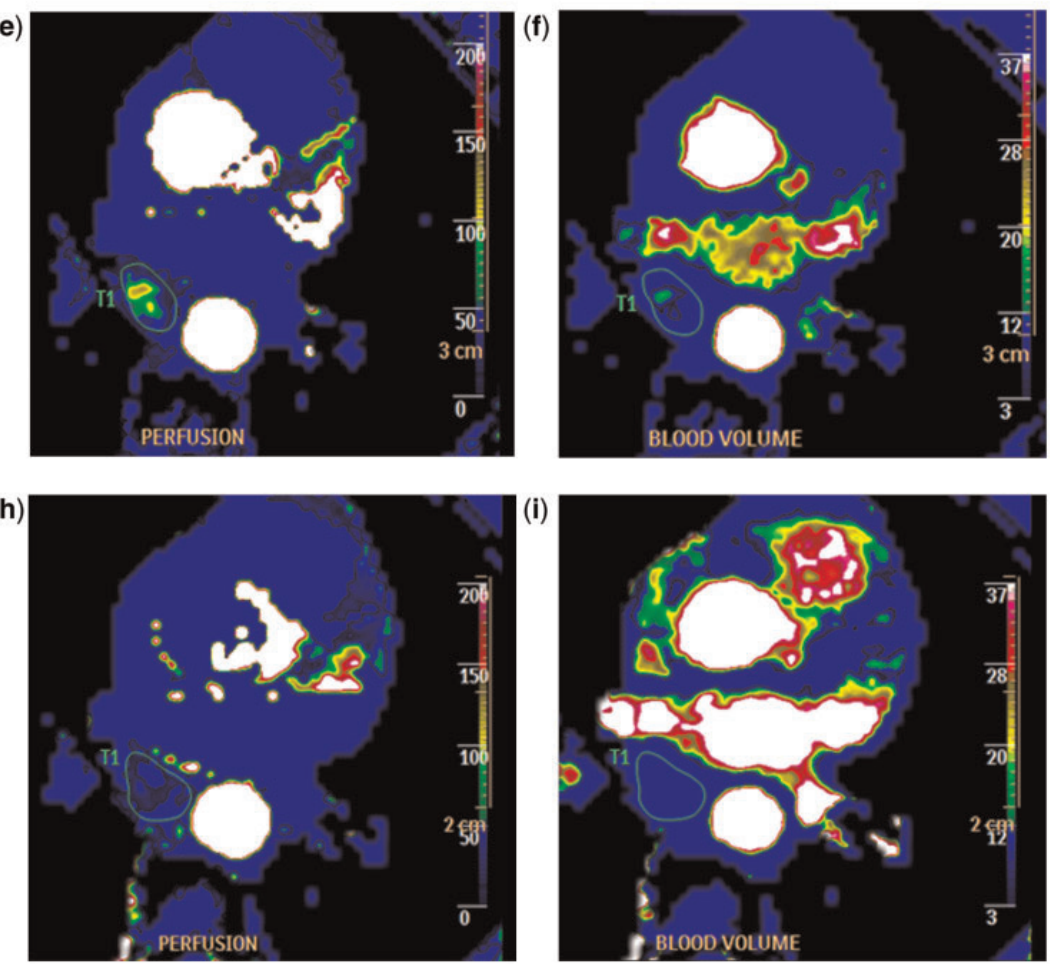

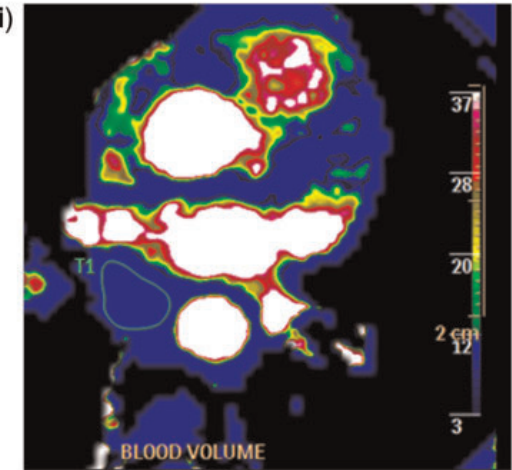

Figure 3 A metastatic lymph node in the mediastinum was a target lesion in this patient with mRCC. In the first column, maximum intensity projection illustrations are presented, the next column illustrates perfusion maps and the third column shows blood volume estimations. The upper row represents baseline, the second row at week 5 and the third row at week 10 after targeted therapy. Visually, the diameter and the volume of the lesion remain unchanged, whereas the enhancement decreased over time. The perfusion and the blood volume also decreased (the green and red colours) within the lesion. Perfusion and blood volume seem to be predictive parameters much earlier than morphological criteria in response evaluation of targeted therapy in mRCC. Quantitative measurements can also be applied.

positrons that decay by the release of photons to produce tomographic images in the PET scanner. The most commonly used radiotracer in oncology is $\left[{ }^{18} \mathrm{~F}\right]$ fluorodeoxyglucose (FDG), an analogue of glucose. Malignant tumour cells metabolize glucose and its analogue FDG. FDG is phosphorylated and trapped in the cell. FDG is excreted by the kidney, which may mask pathologic uptake. In a prospective study, Aide et al. ${ }^{[31]}$ found a high rate of false-negative results when localized RCC was considered (Fig. 4). However, it was found that FDG-PET was efficient for the detection of distant metastasis. In another prospective study ${ }^{[32]}$, the sensitivity of FDG-PET for the detection of distant metastasis from RCC was more modest, mostly due to the small size of the metastasis. In a prospective study of 14 patients with mRCC treated with sunitinib, it was found that PFS correlated with the baseline standardized uptake value ${ }^{[33]}$, and when mRCC shows increased uptake of FDG, PET may play a role in the response evaluation of targeted therapy.

Radiotracers such as $\left[{ }^{11} \mathrm{C}\right]$ acetate, which are not excreted in the urine, may prove useful for imaging RCC. Immuno-PET, which involves radiolabelling of monoclonal antibodies with positron emitters that bind to an antigen on tumour cells ${ }^{[34]}$, may change imaging in RCC. 
(a)

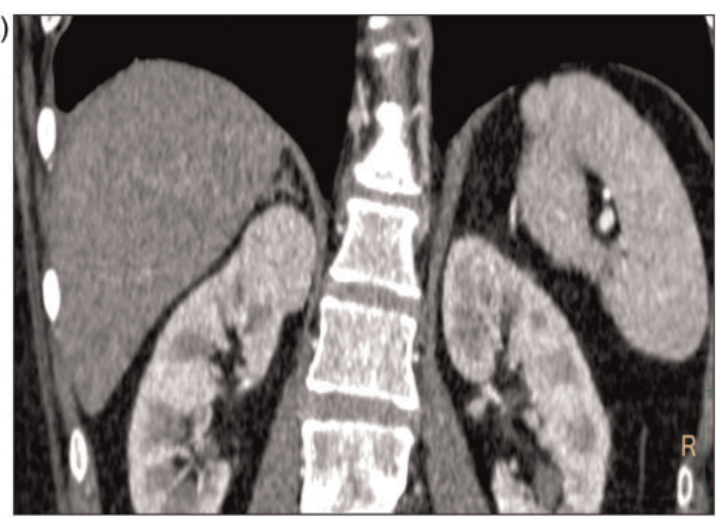

(c)

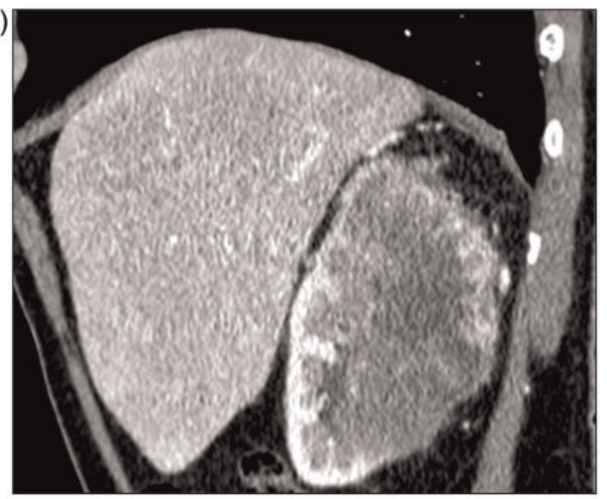

(b)

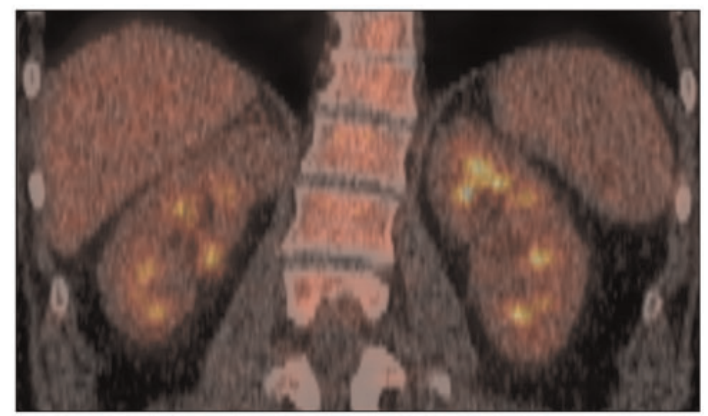

(d)

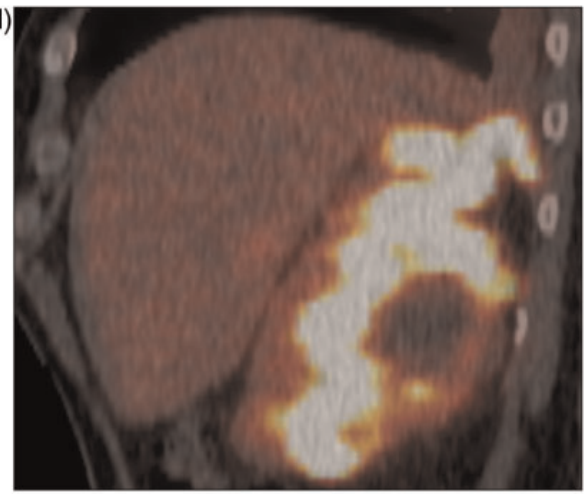

Figure 4 FDG-PET in the evaluation of RCC is hampered by the fact that FDG is excreted by the kidney and may mask FDG uptake in pathologic tissue. Other factors are also involved ${ }^{[31]}$. In (a) a modest contrast enhancement on contrastenhanced CT of the RCC at the upper pole of the kidney is seen. The corresponding PET/CT (b) showed no pathologic uptake of FDG in the tumour. In (c) a large RCC with inhomogeneous contrast enhancement on contrast-enhanced CT is demonstrated. In the corresponding PET/CT (d), a pronounced uptake of FDG in a large part of the tumour is depicted.

\section{Key points}

Historically, patients with mRCC have had a poor prognosis. However, targeted therapies have improved PFS and OS compared with earlier treatment strategies. Traditional imaging evaluation only assesses the morphological tumour changes. Treatment with targeted therapy must often be continued for several months before tumour reduction is seen. No early predictor of successful treatment in patients with mRCC treated with targeted therapies has been established. As targeted therapy causes changes other than tumour size reduction, new follow-up criteria are warranted. In this context, functional imaging surrogate parameters seem to be an improvement and may have potential to enhance traditional imaging evaluation.

\section{Conflict of interest}

The authors have no conflicts of interest to declare.

\section{References}

[1] Ljungberg B, Cowan NC, Hanbury DC, et al. EAU guidelines on renal cell carcinoma: the 2010 update. Eur Urol 2010; 58: 398-406.
[2] Martel CL, Lara PN. Renal cell carcinoma: current status and future directions. Crit Rev Oncol Hematol 2003; 45: $177-190$.

[3] Rini BI, Campbell SC, Escudier B. Renal cell carcinoma. Lancet 2009; 373: 1119-1132.

[4] Chow WH, Devesa SS, Warren JL, Fraumeni Jr JF. Rising incidence of renal cell cancer in the United States. JAMA 1999; 28: 1628-1631.

[5] Diederich S. Imaging beyond RECIST: CT and MRI in molecular therapies. Cancer Imaging 2012; 12: 347-350.

[6] Eisenhauer EA, Therasse P, Bogaerts J, et al. New response evaluation criteria in solid tumours: revised RECIST guideline (version 1.1). Eur J Cancer 2009; 45: 228-247.

[7] Motzer RJ, Hutson TE, Tomczak P, et al. Sunitinib versus interferon alfa in metastatic renal-cell carcinoma. N Engl J Med 2007; 356: $115-124$.

[8] van der Veldt AA, Meijerink MR, van den Eertwegh AJ, Haanen JB, Boven E. Choi response criteria for early prediction of clinical outcome in patients with metastatic renal cell cancer treated with sunitinib. Br J Cancer 2010; 102: 803-809.

[9] Thiam R, Fournier LS, Trinquart L, et al. Optimizing the size variation threshold for the CT evaluation of response in metastatic renal cell carcinoma treated with sunitinib. Ann Oncol 2010; 21: 936-941.

[10] Krajewski KM, Guo M, Van den Abbeele AD, et al. Comparison of four early posttherapy imaging changes (EPTIC; RECIST 1.0, tumor shrinkage, computed tomography tumor density, Choi criteria) in assessing outcome to vascular endothelial growth factortargeted therapy in patients with advanced renal cell carcinoma. Eur Urol 2011; 59: 856-862. 
[11] Wang JH, Min PQ, Wang PJ, et al. Dynamic CT evaluation of tumor vascularity in renal cell carcinoma. Am J Roentgenol 2006; 186: $1423-1430$.

[12] Miles KA, Charnsangavej C, Lee FT, Fishman EK, Horton K, Lee TY. Application of CT in the investigation of angiogenesis in oncology. Acad Radiol 2000; 7: 840-850.

[13] Baccala A, Jr. Hedgepeth R, Kaouk J, Magi-Galluzzi C, Gilligan T, Fergany A. Pathological evidence of necrosis in recurrent renal mass following treatment with sunitinib. Int $\mathrm{J}$ Urol 2007; 14: 1095-1097.

[14] Choi H, Charnsangavej C, Faria SC, et al. Correlation of computed tomography and positron emission tomography in patients with metastatic gastrointestinal stromal tumour treated at a single institution with imatinib mesylate: proposal of new computed tomography response criteria. J Clin Oncol 2007; 25: 1753-1759.

[15] Hittinger M, Staehler M, Schramm N, et al. Course of size and density of metastatic renal cell carcinoma lesions in the early follow-up of molecular targeted therapy. Urol Oncol 2012; 30: 695-703.

[16] Nathan PD, Vinayan A, Stott D, Juttla J, Goh V. CT response assessment combining reduction in both size and arterial phase density correlates with time to progression in metastatic renal cancer patients treated with targeted therapies. Cancer Biol Ther 2010; 9: 15-19.

[17] Han KS, Jung DC, Choi HJ, et al. Pretreatment assessment of tumor enhancement on contrast-enhanced computed tomography as a potential predictor of treatment outcome in metastatic renal cell carcinoma patients receiving antiangiogenic therapy. Cancer 2010; 116: 2332-2342.

[18] Smith AD, Lieber ML, Shah SN. Assessing tumor response and detecting recurrence in metastatic renal cell carcinoma on targeted therapy: importance of size and attenuation on contrastenhanced CT. AJR Am J Roentgenol 2010; 194: 157-165.

[19] Smith AD, Shah SN, Rini BI, Lieber ML, Remer EM. Morphology, Attenuation, Size, and Structure (MASS) criteria: assessing response and predicting clinical outcome in metastatic renal cell carcinoma on antiangiogenic targeted therapy. AJR Am J Roentgenol 2010; 194: 1470-1478. doi:10.2214/AJR.09.3456. PubMed PMID: 20489085.

[20] Goh V, Ganeshan B, Nathan P, Juttla JK, Vinayan A, Miles KA. Assessment of response to tyrosine kinase inhibitors in metastatic renal cell cancer: CT texture as a predictive biomarker. Radiology 2011; 261: 165-171.

[21] Reiner CS, Roessle M, Thiesler T, et al. Computed tomography perfusion imaging of renal cell carcinoma: systematic comparison with histopathological angiogenic and prognostic markers. Invest Radiol 2013; 48: 183-191.

[22] Chen Y, Zhang J, Dai J, Feng X, Lu H, Zhou C. Angiogenesis of renal cell carcinoma: perfusion $\mathrm{CT}$ findings. Abdom Imaging 2010; 35: 622-628.
[23] Fournier LS, Oudard S, Thiam R, et al. Metastatic renal carcinoma: evaluation of antiangiogenic therapy with dynamic contrast-enhanced CT. Radiology 2010; 256: 511-518.

[24] Ng CS, Wang X, Faria SC, Lin E, Charnsangavej C, Tannir NM. Perfusion CT in patients with metastatic renal cell carcinoma treated with interferon. AJR Am J Roentgenol 2010; 194: 166-171.

[25] Miles KA, Lee TY, Goh V, et al. Current status and guidelines for the assessment of tumour vascular support with dynamic contrast-enhanced computed tomography. Eur Radiol 2012; 22: 1430-1441.

[26] Flaherty KT, Rosen MA, Heitjan DF, et al. Pilot study of DCEMRI to predict progression-free survival with sorafenib therapy in renal cell carcinoma. Cancer Biol Ther 2008; 7: 496-501.

[27] Hahn OM, Yang C, Medved M, et al. Dynamic contrast-enhanced magnetic resonance imaging pharmacodynamic biomarker study of sorafenib in metastatic renal carcinoma. J Clin Oncol 2008; 26: $4572-4578$.

[28] Taouli B, Thakur RK, Mannelli L, et al. Renal lesions: characterization with diffusion-weighted imaging versus contrast-enhanced MR imaging. Radiology 2009; 251: 398-407.

[29] Desar IM, ter Voert EG, Hambrock T, et al. Functional MRI techniques demonstrate early vascular changes in renal cell cancer patients treated with sunitinib: a pilot study. Cancer Imaging 2012; 11: 259-265.

[30] Lassau N, Koscielny S, Albiges L, et al. Metastatic renal cell carcinoma treated with sunitinib: early evaluation of treatment response using dynamic contrast-enhanced ultrasonography. Clin Cancer Res 2010; 16: 1216-1225.

[31] Aide N, Cappele O, Bottet P, et al. Efficiency of [(18)F]FDG PET in characterising renal cancer and detecting distant metastases: a comparison with CT. Eur J Nucl Med Mol Imaging 2003; 30: 1236-1245.

[32] Majhail NS, Urbain JL, Albani JM, et al. F-18 fluorodeoxyglucose positron emission tomography in the evaluation of distant metastases from renal cell carcinoma. J Clin Oncol 2003; 21: 3995-4000.

[33] Revheim ME, Winge-Main AK, Hagen G, Fjeld JG, Fosså SD, Lilleby W. Combined positron emission tomography/computed tomography in sunitinib therapy assessment of patients with metastatic renal cell carcinoma. Clin Oncol (R Coll Radiol) 2011339-343.

[34] Divgi CR, Pandit-Taskar N, Jungbluth AA, et al. Preoperative characterisation of clear-cell renal carcinoma using iodine-124labelled antibody chimeric G250 (124I-cG250) and PET in patients with renal masses: a phase I trial. Lancet Oncol 2007; 8: 304-310. 